\title{
Correction to: Oral Health in Older Adults with Dementia
}

\author{
Maryam Tabrizi, Victoria A. Mañón, and Clark Whitmire
}

\section{Correction to:}

A. G. Catic (ed.), Dementia and Chronic Disease, https://doi.org/10.1007/978-3-030-46398-4_13

The book was inadvertently published with an incorrect title of Chapter 13 in Table of contents and Chapter 13. The title is now corrected as "Oral Health in Older Adults with Dementia" in the book. 